\title{
L'incorporation d'éléments de la musique baroque dans le tango d'Astor Piazzolla
}

\section{Thomas Fontes Saboga Cardoso}

Résumé: Nous scrutons de façon particulière dans cet article la combinaison entre tango et musique baroque proposée par le compositeur et bandonéoniste argentin Astor Piazzolla (1921-1992). Les traits du tango sont privilégiés dans cette articulation et constituent les éléments de base repérables principalement par des gestes mélodiques, des modèles d'accompagnement et des timbres caractéristiques très fréquemment employés, qui assurent aussi bien la cohésion et l'unité musicale que l'identité du tango. Nous observerons ensuite une autre stratégie employée par le compositeur, consistant à altérer tout au long d'une même pièce le degré d'intensité des courants combinés - métissage avec changement de lecture , ce qui met en valeur de cette façon cette articulation elle-même. Nous verrons également comment Piazzolla dose avec une très grande précision et justesse les divers ingrédients de ces univers musicaux.

Mots clé : Astor Piazzolla; tango; musique baroque; jazz; métissage musical.

Abstract: The aim of the present article is to understand the articulation of tango and baroque music in the production of the composer and bandoneonist Astor Piazzolla (1921-1992). Tango features are favored in this junction, constituting the basic elements mainly through the presence of melodic gestures, accompaniment models and characteristic timbres very frequently used, which assure the cohesion and musical unity as well as the identity of the tango. Another blending strategy is considered, in which Piazzolla changes the degree of intensity of these musical streams through one same piece, and thus valuing the articulation itself. We will also give attention to the just and precise amount of the diverse ingredients from these musical streams employed by the composer.

Keywords: Astor Piazzolla; tango; baroque music; jazz; musical articulation. 


\section{Introduction}

Nous nous proposons dans cet article de comprendre comment le compositeur et bandonéoniste argentin Astor Piazzolla (1921-1992) intègre certains traits de la musique baroque dans sa production musicale, qui trouve dans la musique de tango sa base structurelle. Comment articule-t-il ces univers musicaux ? Quelles sont ses stratégies pour parvenir à une unité musicale ?

Afin de pouvoir répondre à ces questions, notre méthodologie analytique a consisté, dans un premier moment, à discerner et identifier de quels courants musicaux tirent leur origine les diverses caractéristiques de sa musique. Ce travail a été réalisé à travers l'étude de quatre grands groupes de données: enregistrements et partitions de Piazzolla entre 1955 et 1965, période d'établissement et de mûrissement de son style personnel (Brunelli, 1992, p. 210-211 ; Pelinski, 2008, p. 38 ; Fischerman et Gilbert, 2009, p. 178) ; études musicologiques portant sur son œuvre ; données biographiques du compositeur; et matériels concernant les genres musicaux qu'il articule. Dans un deuxième moment, nous avons essayé de comprendre quelle était la dynamique mise en œuvre dans l'articulation de ces diverses caractéristiques du baroque et du tango, et trois résultats seront ici proposés. Nous commencerons par observer comment les éléments de la musique baroque sont généralement accompagnés de plusieurs éléments du tango, ce processus étant donc réalisé dans une atmosphère de ce genre, dans une vraisemblable « expansion du tango» (Mauriño, 2008, p. 31). Il s'agit ainsi d'une stratégie capable en même temps de donner une unité musicale à sa production sonore et de préserver l'identité du tango. Par la suite, nous verrons comment ce musicien utilise à certaines occasions une stratégie particulière de métissage, qui consiste à altérer le degré d'intensité des courants musicaux articulés tout au long d'une même composition, et que 
nous avons appelée métissage avec changement de clé de lecture'. Dans un dernier moment, on observera comment ce compositeur dose avec une grande précision les divers ingrédients utilisés dans cette articulation.

Les rapports de Piazzolla avec la musique baroque ont déjà fait l'objet d'études musicologiques. Sonia López (2008) avait déjà proposé l'expression «double interprétation analytique » sur l'exposition de la fugue de Fuga y misterio, en démontrant que son thème correspondait aussi bien aux exigences d'un sujet de fugue qu'aux caractéristiques mélodiques du tango, en gardant ainsi une cohérence pour ces deux cultures. Ramón Pelinski (2008) propose l'expression « changement de clé de lecture », que nous reprenons en détail dans ce travail. Gabriela Goldenberg (2003), en scrutant les liens avec la fugue, découvre la raison de l'utilisation exclusive de l'exposition par Piazzolla en dévoilant sa fonction : l'introduction progressive des éléments dans l'exposition de la fugue offre à sa musique une croissance graduelle de la densité sonore, élément très recherché par Piazzolla. En voyant dans ces travaux féconds une importante base sur laquelle bâtir notre recherche, nous nous proposons donc dans cet article de poursuivre cette perspective d'études déjà entamée. Nous devons également aux études d'Omar Brunelli l'idée que les éléments exogènes au tango sont incorporés « à la trame de son écriture de telle sorte qu'ils s'entrelacent pour pratiquement disparaître absorbés dans le langage tanguistique $»^{2}$, suivie par la présente recherche, comme l'atteste le titre de cette contribution.

Observons pour commencer quelques brèves considérations sur les rapports historiques de Piazzolla avec cette période historique.

\footnotetext{
1 Notion s'inspirant de Ramón Pelinski (2008, p. 46).

2 «[...] los [los rasgos jazzísticos] incorpora a la trama de su escritura musical de modo tal que se entrelazan [...] para prácticamente desaparecer absorbidos en el lenguaje tanguístico.» Brunelli (2011). Toutes les traductions sont de notre responsabilité.
} 


\section{Le baroque dans le tango de Piazzolla}

Piazzolla exprime dans ses déclarations une admiration ancienne et profonde pour la musique de Jean-Sébastien Bach (Gorin, 1990, p. 117 ; Piazzolla, Diana, 2002, p. 74). Il affirme également avoir étudié certains éléments caractéristiques du baroque, comme le contrepoint et la fugue, avec ses maîtres Alberto Ginastera (Speratti, 1969, p. 59) et Nadia Boulanger (Piazzolla, Diana, 2002, p. 142 ; Saavedra, 1989). Voyons à présent comment il les incorpore dans sa production sonore.

Figure 1 - Homero Manzi et Sebastián Piana. « Milonga triste ». Version d'Astor Piazzolla interprétée par lui et son Quintette « Nuevo Tango » dans l'album Nuestro tiempo (2005). De 2'29 à 2'39, mesure à 4/4, $\pm \cong 100$, transcription approximative de l'auteur du présent article.

Guitare électrique

Contrebasse

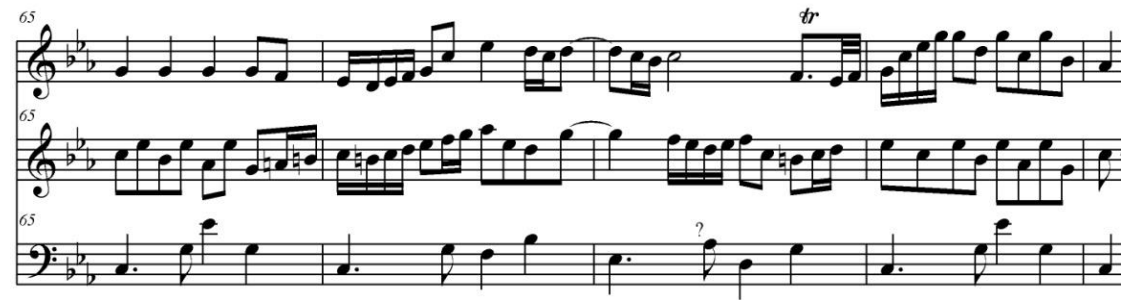

Dans ce passage, on identifie la présence d'une polyphonie imitative entre violon et guitare électrique, observable dans le dialogue suscité par les motifs rythmiques de quatre doubles-croches (jouées en même temps par les deux instruments au premier temps de la mesure 66, par la guitare électrique au deuxième temps de la mesure 67, et par le violon au premier temps de la mesure 68), le motif dactyle (à la guitare électrique au quatrième temps de la mesure 65 et 67 et deuxième de la mesure 66, et au violon avec variation au premier temps de la mesure 67, avec variation en anapeste un temps avant, et avec un trille et une accélération rythmique au dernier temps de la mesure 67), ainsi que par les croches, qui alternent entre les deux voix exposées.

On y observe également la présence d'un geste conclusif au violon entre les mesures 67 et 68 : un trille est suivi d'une broderie inférieure qui 
se résout ensuite sur la note supérieure, proposant ainsi un rapport avec la musique baroque par le phrasé fortement ornementé.

II est aussi possible de comprendre la mélodie avec une pédale à la note supérieure (à la guitare électrique aux m. 65 et 68 , en ayant la note mià la pédale supérieure ; au violon à la $\mathrm{m}$. 68 , la note so/étant à la pédale supérieure) comme proposant un lien supplémentaire avec le baroque ${ }^{3}$. II est ainsi possible de remarquer une similarité avec, par exemple, le sujet de la fugue de la Toccata et Fugue en ré mineur de J.-S. Bach (s.d.) et sa construction similaire avec une pédale à la note supérieure.

En même temps, la contrebasse qui se superpose à cette écriture

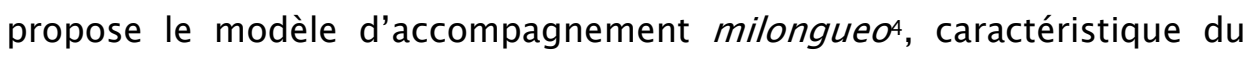
tango. On constate ainsi l'existence d'une articulation avec cette écriture baroque, qui s'effectue par-dessus un modèle d'accompagnement caractéristique du genre populaire urbain.

Dans cet exemple les rapports entre baroque et tango, peuvent être encore davantage approfondis. Ce morceau, intitulé Milonga triste, est une composition de Sebastián Piana et Homero Manzi, compositeurs de tango, et cet arrangement de Piazzolla est chanté par Hector de Rosas - ce passage est un bref interlude instrumental. On se trouve ainsi devant une composition de tango, interprétée par un chanteur de ce genre. Dans ce cas précis, étant donné qu'il s'agit d'un arrangement d'une chanson émanant de l'univers même du tango, l'articulation entre les éléments dévoile plus clairement la base de ce genre, les éléments de la musique

\footnotetext{
3 Un autre élément qu'il convient de retenir en ce qui concerne ce rapprochement avec le baroque, figure dans un texte du compositeur lui-même sur la pochette de cet album, dans lequel il affirme avoir dans ce morceau « senti la nécessité de faire un arrangement de chambre », et aussi " avoir voulu créer l'atmosphère de toute l'œuvre dans un climat médiéval », où l'on perçoit l'intention de proposer un dialogue avec la musique savante («musique de chambre ») et ancienne ( médiévale »). "Sentí la necesidad de hacer un arreglo de cámara. " "Quise ambientar toda la obra en un clima medieval [...] » Astor Piazzolla (2005).

4 Le milongueo est un modèle d'accompagnement caractéristique du tango, dans lequel la basse propose, sur une mesure à $4 / 4$, une noire pointée suivie d'une croche et de deux noires. Peralta $(2012$, p. 85).
} 
baroque étant alors introduits dans cet univers, qui demeure ainsi central dans ce métissage ${ }^{5}$. Examinons un autre cas.

Figure 2 - Astor Piazzolla. Resurrección del ánge/ (2002). Bandonéon, violon, guitare électrique et contrebasse, $R 2-R 2+6$, mesure à $4 / 4$,

Tango, Andante Moderato $( \pm=92)$

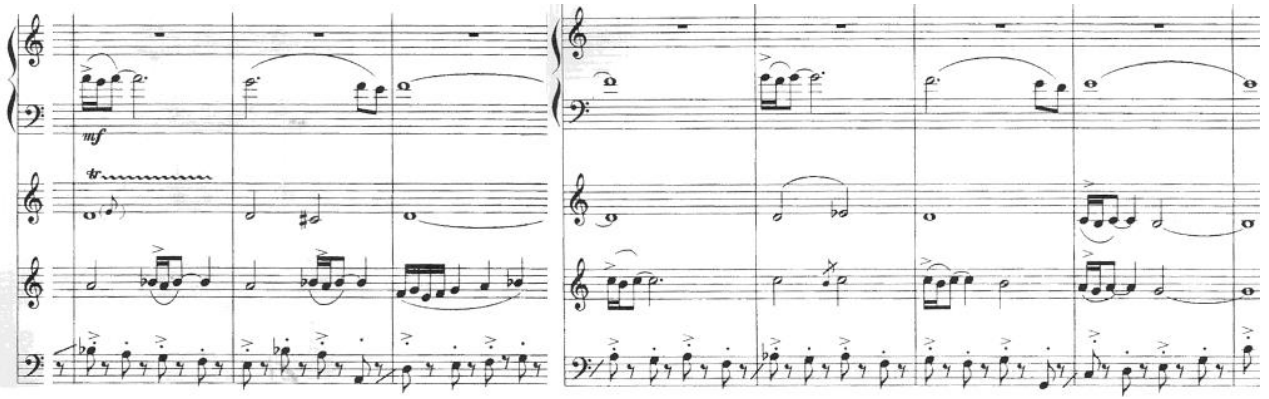

À la contrebasse, on identifie un modèle d'accompagnement caractéristique du tango, le marcato en uno tres6, avec l'écriture d'arrastres7, élément distinctif du genre, afin de donner encore plus d'emphase au swing du tango. Par-dessus, on perçoit une texture contrapuntique, avec notamment des imitations fréquentes du motif de broderie entre le bandonéon et la guitare électriques. L'utilisation d'une

5 Le rapport entre le tango et le baroque dans la musique de Piazzolla avait déjà aussi été proposé par Clemente, qui affirme que le compositeur est « capable de mélanger de façon réussie le langage motivique du tango traditionnel avec les principes de conduction de voix du dix-huitième siècle. " « Through such contexts Piazzolla is able to successfully blend the traditional tango motivic langage with eighteenth century voice leading principles. »Clemente (2012, p. 38).

6 Le marcato en uno tres est un modèle d'accompagnement caractéristique du tango, dans lequel la basse marque les quatre temps de la mesure quaternaire en staccato, en accentuant le premier et le troisième temps. Marsili (2012, p. 47-48) ; Peralta (2012, p. 56).

7 L'arrastre est un élément distinctif du tango, qui contribue à produire le swing caractéristique de ce genre. Proposé plus fréquemment à la contrebasse, il se caractérise par "l'accentuation typique du quatrième temps suivi d'un glissando traînant ascendant ou descendant fortement marqué vers le premier temps de la mesure suivante. » (Marsili, 2012, p. 56) et peut être indiqué par une ligne oblique (Salgán, 2012, p. 86).

8 Ce motif de broderie est proposé par le bandonéon au premier temps, et ensuite repris par la guitare électrique au troisième temps des première et deuxième mesures proposées. II sera repris aux premiers temps des quatre dernières mesures, dans l'ordre suivant : à la guitare électrique, au bandonéon, à la guitare électrique, puis à la guitare électrique doublée par le violon. Cette description n'a 
augmentation rythmique de cette broderie, à la suite de la mélodie au bandonéon ${ }^{9}$, est aussi caractéristique de la technique du contrepoint imitatif10; les articulations mélodiques qui évitent soigneusement de coïncider dans le temps le sont également. On peut citer comme éléments moins déterminants, mais qui semblent également suggérer encore une référence à cette sonorité baroque, la broderie tronquée au premier temps de la troisième mesure à la guitare électrique, l'écriture d'une appoggiature sur ce même instrument à la sixième mesure, et la présence d'un trille au violon à la première mesure présentée. On constate alors la présence d'une texture de contrepoint imitatif, caractéristique de la musique baroque, réalisée par-dessus une basse, qui donne la base du morceau, construite sur un modèle d'accompagnement caractéristique du tango, et l'on perçoit ainsi comment le compositeur se propose d'introduire ces éléments baroques dans une ambiance de tango, en cherchant de cette façon tout à la fois à produire une unité musicale et à préserver l'identité de ce genre.

On retrouve cette même façon d'articuler ces éléments dans la version de Piazzolla de Recuerdo de la Bohemia / Milonguita, d'Enrique Delfino, Manuel Romero et Samuel Linning (2005), de 1'02 à 1'12, quand une polyphonie imitative est proposée sur un rythme 3-3-211 caractéristique du tango, et ce sur un arrangement de morceaux créés par des compositeurs de ce genre interprétés par un chanteur de tango; ou dans les premiers instants de Sin retorno, d'Alberto Coronato (2005), quand une polyphonie imitative est réalisée sur une basse qui fait

pas pris en compte les transformations proposées pour cette broderie, comme l'augmentation rythmique au bandonéon (observez les croches aux deuxième et cinquième mesures) ou l'ajout d'une note, qui donne naissance à une broderie tronquée à la guitare électrique (voir troisième mesure).

9 Observer l'anacrouse en croches des troisième et septième mesures présentées. 10 Les blanches au violon aux deuxième et cinquième mesures présentées peuvent aussi être comprises comme une imitation par augmentation de ces broderies mélodiques, s'ajoutant ainsi à ce tissu polyphonique.

11 Ce modèle d'accompagnement caractéristique du tango (Pelinski, 2008, p. 43 ; Marsili, 2012, p. 80) consiste à accentuer, dans une mesure à $4 / 4$, les première, quatrième et septième croches. 
référence au marcato en marquant les quatre temps de la mesure et étant réalisée par une doublure de la contrebasse avec la main gauche du bandonéon. Piazzolla peut suggérer d'autres façons de réaliser cette articulation en proposant, par exemple, une strette avec un motif de tango, comme dans les six dernières mesures de Mar del Plata 70 (1974). Considérons à présent une stratégie supplémentaire employée pour combiner ces univers sonores.

\section{Le métissage avec changement de clé de lecture}

Un extrait de Ramón Pelinski nous introduit dans ce type de métissage.

[...] La construction du sujet est soumise à un procès de défamiliarisation à travers une double asymétrie : celle qui provient de son articulation tanguistique à l'intérieur de la mesure. Et celle qui provient de la superposition au sujet «baroque » d'une partie percussive en valeurs rythmiques asymétriques. Dans les deux cas, la configuration rythmique du sujet est soumise au tango à travers les syncopes et déplacements asymétriques provoqués par des accents dynamiques étrangers à la pratique baroque historique. Et qui plus est, dans les deux cas l'énonciation du sujet [de fugue] à l'unisson [après l'exposition de la fugue], sans les contre-sujets, sur une formule réitérative de la basse, nous invite à une audition ambiguë : analogiquement à la vision double de la bouteille de Klein, on pourrait écouter cette résignification du sujet indistinctement soit en clé «baroque », soit en clé de tango. Ou encore mieux: simplement comme musique de Piazzolla.12

Dans cette analyse très fertile, Pelinski dévoile l'idée d'une double lecture : Iorsque l'on écoute le sujet pendant l'exposition de la fugue, on est invité à une écoute en clé baroque, et quand on l'écoute en

12 «[...] la enunciación del sujeto al unísono, sin los contrasujetos, sobre una fórmula reiterativa del bajo, invita a una audición ambigua: analógicamente a la visión doble del vaso de Klein, podría escucharse esta resignificación del sujeto indistintamente sea en clave "barroca", sea en clave de tango. O quizás mejor: simplemente como música de Piazzolla. »Pelinski, 2008, p. 46. Nous avons traduit l'expression vaso de Klein par «bouteille de Klein ». 
homorythmie, c'est la clé de tango qui est alors suggérée 13. Observons deux passages de Muerte del ángel afin de mieux comprendre cette proposition.

Figure 3 - Astor Piazzolla. Muerte del ánge/ (s.d.). Exposition de la fugue, mesures 1-23 [m. 7-29 du manuscrit de José Bragato], $\pm \cong 142$, vers 0 '00, synthèse du quintette réalisée à partir du manuscrit de Bragato et de la transcription approximative de l'auteur
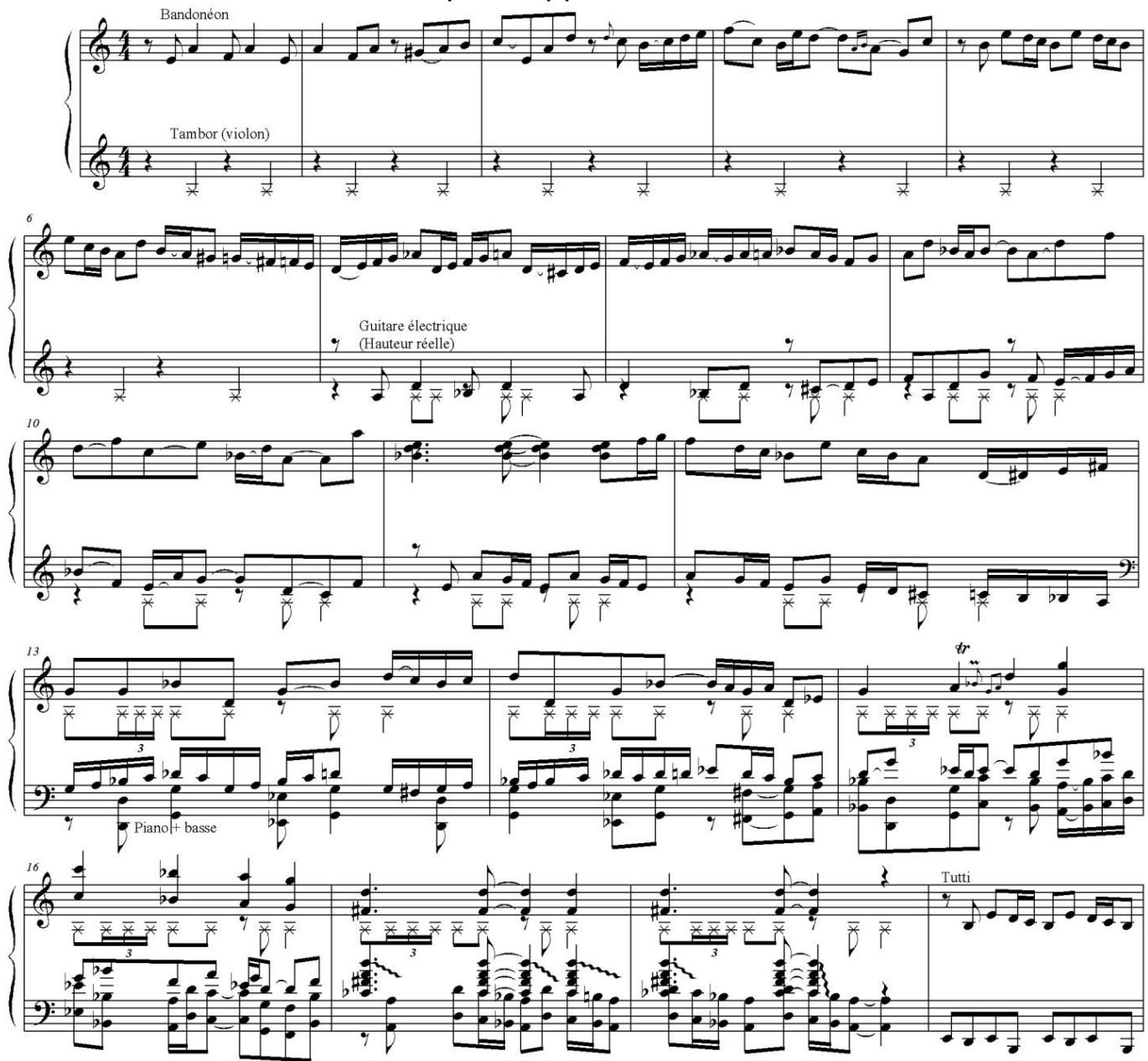

13 Si Pelinski semble proposer également l'idée que cette résignification peut être réalisée lors d'un même passage, le thème lui-même pouvant être écouté soit comme une phrase de tango soit comme un sujet de fugue baroque, nous proposons d'utiliser cet intéressant concept de «clé de lecture » pour signaler le changement proposé par un traitement musical différent d'un même matériau dans deux passages d'une même composition. 
Figure 4 - Astor Piazzolla. Muerte del ángel(s. d.). Thème traité de façon homorythmique, m. 21-29, $\cong 1140$, vers 0 '34, transcription des mesures 27-35 du manuscrit de Bragato et synthèse du quintette, mesure à $4 / 4$

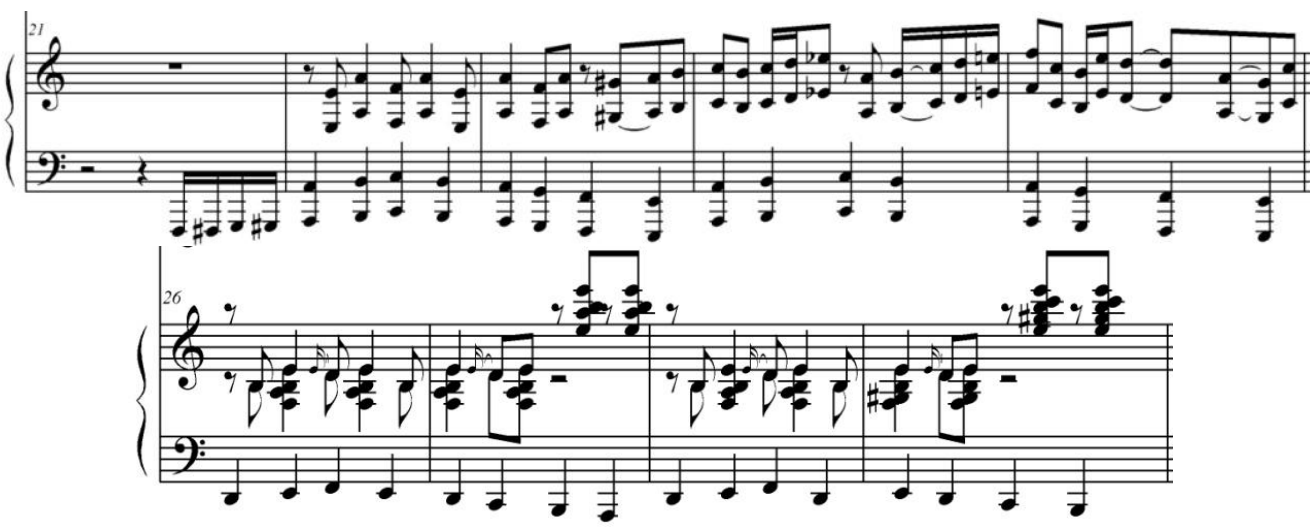

Ces illustrations permettent de comprendre les objectifs de Pelinski. On voit d'abord l'exposition de la fugue, avec une texture contrapuntique, les entrées du sujet s'effectuant aux mesures 1, 7 et 13, avec la présence d'un contre-sujet aux deuxième et troisième apparitions du thème. Dans l'extrait suivant, on retrouve la même phrase musicale du sujet, avec quelques petites variations, mais sur une texture sonore complètement différente. Elle est en effet désormais seulement accompagnée par une basse, qui propose un accompagnement caractéristique de tango, le marcato en uno tres ${ }^{14}$, avec une variante de levée chromatique typique ${ }^{15}$ qui introduit le swing du genre, et ce en étant réalisé par une doublure de la contrebasse et de la main gauche du piano, élément également distinctif du tango ${ }^{16}$, la guitare électrique s'ajoutant

14 La partition signée par José Bragato signale, à la contrebasse, l'usage d'un archet, avec le premier et le troisième temps accentués et en staccato, le deuxième et le quatrième en staccato.

15 La levée chromatique typique est un élément caractéristique de la basse du tango. Sur une mesure à $4 / 4$, elle consiste en une croche placée sur le troisième contretemps, suivie de quatre doubles-croches, en un mouvement chromatique ascendant aboutissant au premier temps de la mesure suivante. Marsili (2012, p. 56-57).

16 Il est d'usage dans le tango de doubler la basse, la contrebasse et la main gauche de piano jouant à l'unisson. Peralta (2012, p. 56) ; Salgán (2012, p. 84) ; Marsili (2012, p. 263). 
encore à cette doublure. Piazzolla propose ainsi une même phrase musicale en clé baroque, pendant l'exposition de la fugue, afin de suggérer une interprétation en clé de tango dans la suite de la composition. Dans cette façon de concevoir la pensée musicale de Piazzolla, le compositeur joue avec ces clés, en proposant des lectures distinctes pour le même matériau : c'est cette façon particulière d'articuler ces deux univers musicaux que nous comprenons comme un métissage avec un changement de clé de lecture. Voyons un autre cas.

Figure 5 - Astor Piazzolla. La mufa (1974), m. 1-28, Tango, $\pm \cong 100$, vers 0 '00
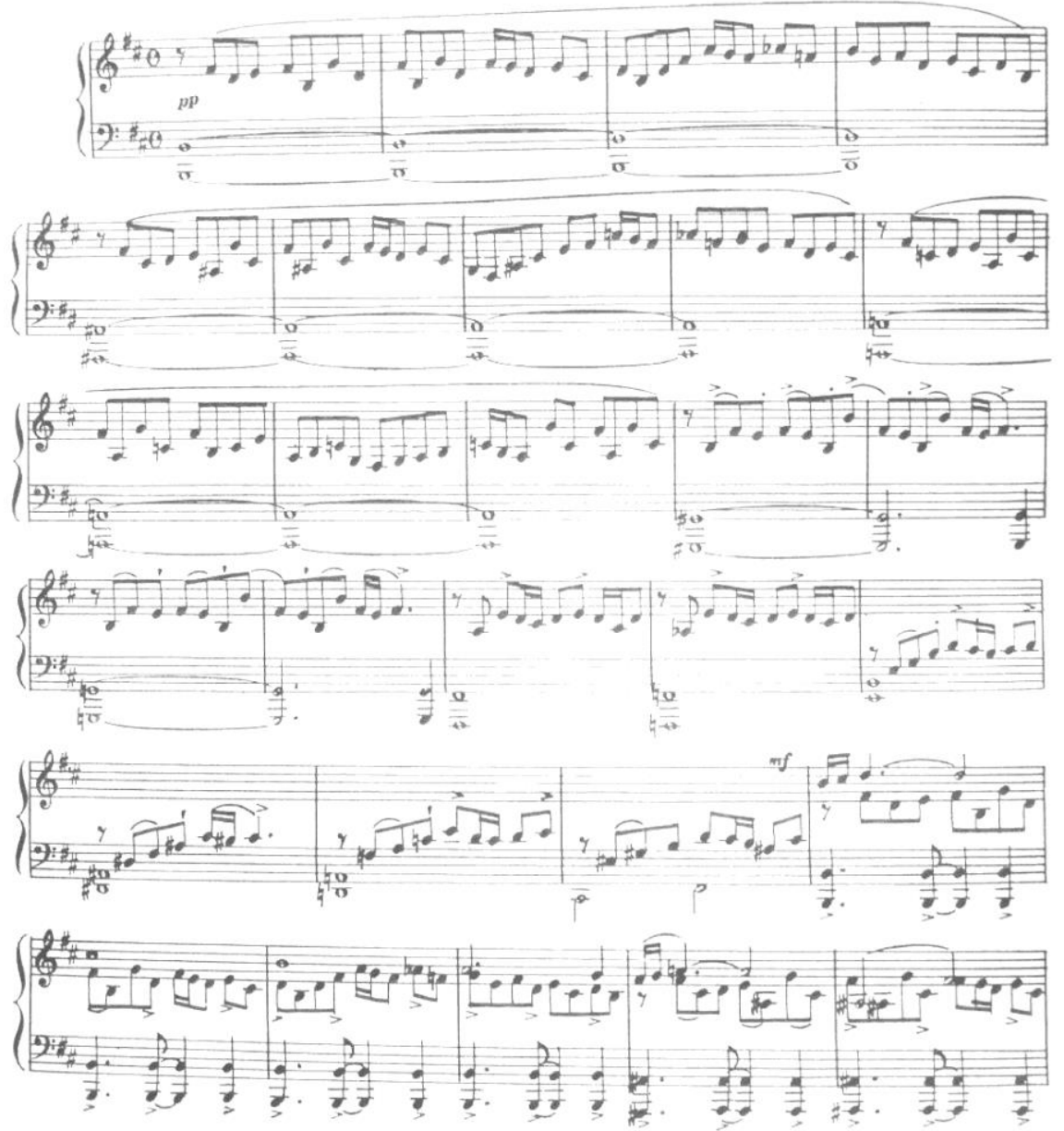
Aux premières mesures de cet exemple, on identifie une mélodie acéphale, avec la présence, par la suite, d'un rythme anapeste, et sur une basse statique. Jusqu'à la mesure 12 , les indications phraséologiques proposent une mélodie entièrement jouée en legato. Ensuite, les indications phraséologiques ajoutées proposent clairement l'interprétation d'un geste caractéristique du tango 17, observable aux mesures 13 et 1418. L'audition de ce passage interprété par le groupe de Piazzolla (2005) s'avère très intéressante de par sa capacité à démontrer de façon effective la différence entre les deux façons de phraser, et permet de constater la signification importante du geste musical proposé dans cette façon de noter les indications phraséologiques. On se trouve alors devant un autre exemple de changement de clé de lecture, les indications phraséologiques étant dans le cas présent la clé, qui propose par le biais de son caractère legato aux premières mesures une sonorité plutôt en rapport avec la musique baroque, avec de surcroît la sonorité de l'orgue suggérée par le bandonéon 19, et au moyen de son articulation de tango,

17 Selon Pelinski (2008, p. 48), ce sont en grande partie les modalités caractéristiques de l'interprétation qui transforment le résultat sonore en tango. Parmi ces modalités, le phrasé (Marsili, 2012, p. 39-40 ; Salgán, 2012, p. 59) constitue un élément important. Marsili (2012, p. 54-55) décrit les traits distinctifs des phrases de tango: motifs asymétriques; motifs en syncope; motifs acéphales; et accents sur le contretemps. A partir de l'analyse des principales caractéristiques mélodiques de ce genre appliquées à la musique de Piazzola, nous proposons de considérer la phraséologie particulière suivante, souvent employée par Piazzolla, et fréquemment soigneusement notée par le compositeur, comme une phraséologie caractéristique du tango. Nous la décrirons ici de façon schématique, en sachant qu'il faut prendre en compte l'existence de variations. En mesure à $4 / 4$, il s'agit d'une phrase acéphale, qui démarre sur le premier contretemps, de façon accentuée ; cette première note, une croche, présente une liaison vers la croche qui suit ; une troisième croche lui succède, sur le deuxième contretemps, donc, notée en staccato ; la note suivante, sur le troisième temps, est souvent accentuée, ce qui génère une mélodie asymétrique. II est très courant de trouver dans la suite de la mélodie des rythmes anapestes ou dactyles, rythmes notés que nous comprenons comme une dérivation des irrégularités rythmiques des interprétations vocales de tango (Kutnowski, 2008, p. 102).

18 II convient d'observer la première note acéphale accentuée, la présence d'un lié depuis celle-ci vers la deuxième croche, la troisième en staccato, et la quatrième accentuée.

19 Le legato étant privilégié, cette mélodie ainsi réalisée au bandonéon propose un puissant rapport avec la sonorité de l'orgue, instrument caractéristique du baroque. Par ailleurs, cet instrument fut «inventé en Allemagne pour remplacer l'orgue dans les cérémonies religieuses à l'extérieur ou dans des petites églises ». 
une sonorité dans l'esthétique de ce genre musical20. Analysons un autre cas.

Figure 6 - Astor Piazzolla. Resurrección del ángel (2002). Violon, guitare et contrebasse, $\mathrm{R} 1-\mathrm{R} 1+4$, mesure à 4/4, Tango, Andante Moderato

$$
( \pm=92)
$$
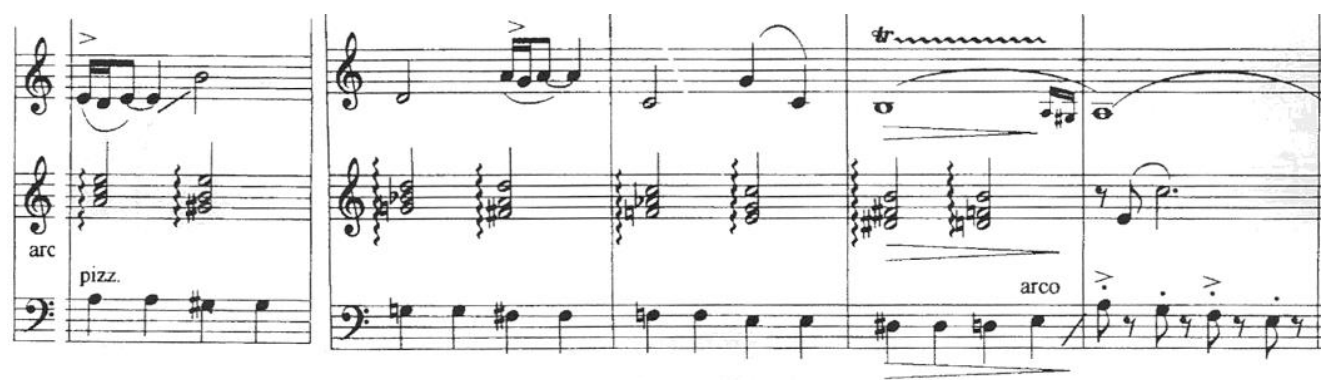

Figure 7 - Astor Piazzolla. Resurrección del ángel (2002). Quintette, R14+1 - R14+5, Tango, Tempo I, $\pm=92$

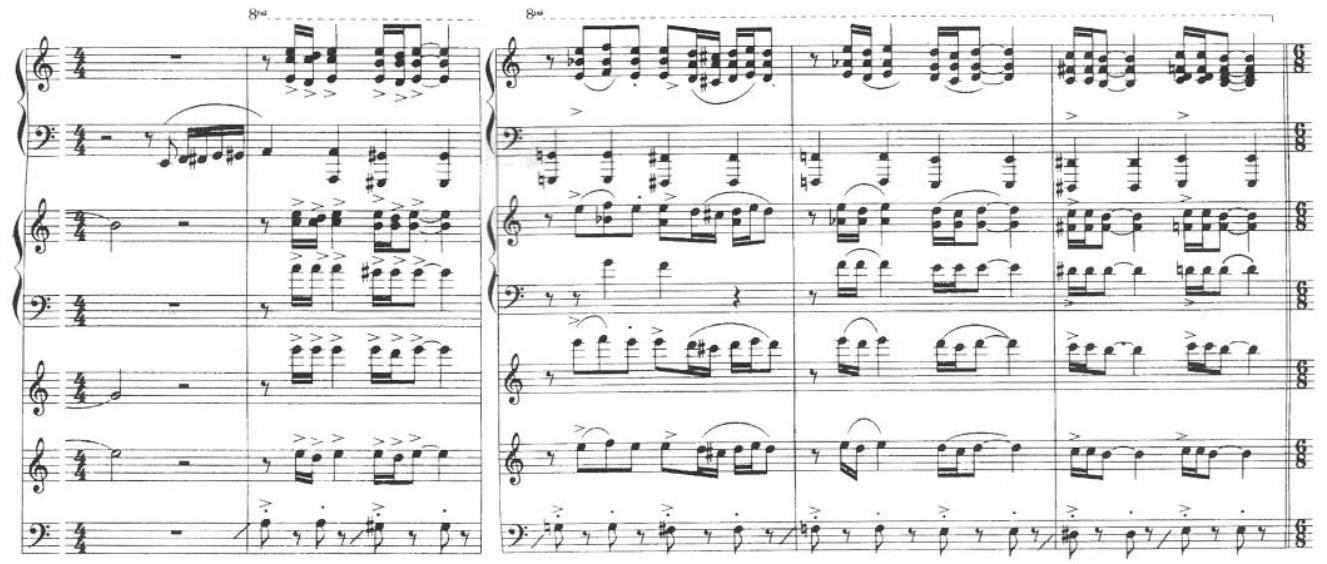

« Inventado en Alemania para reemplazar el órgano en las celebraciones religiosas al aire libre o en pequeñas iglesias [...]. » Mauriño, op. cit., p. 22.

20 La suite de ce passage montre que cette expression est vraiment adaptée à ce morceau : on trouve, à la reprise à la mesure 23 de ce même exemple, la même mélodie qu'au début, mais, cette fois, proposée sur un accompagnement en 3-32 , qui va encore une fois beaucoup modifier tout à la fois l'interprétation et la perception de cette mélodie. La même chose se produit quand le même thème est proposé en même temps qu'un parallélisme particulièrement dense et complexe à la mesure 92, également sur un rythme 3-3-2: Piazzolla semble alors proposer plusieurs clés de lecture de ce thème. 
Nous allons à présent observer le contraste entre les traitements réservés aux deux derniers extraits proposés du même morceau, tous deux construits rigoureusement sur la même harmonie et un motif mélodique similaire. Dans le premier exemple, il est possible d'observer un traitement sobre: une basse en noires, qui peut aussi bien faire référence à la Gehende Bässe du baroque dont parle Pelinski (2008, p. 47), qu'aux modèles d'accompagnement du tango qui marquent les quatre temps de la mesure, avec des accords en blanches à la guitare électrique. Le solo de violon qui est superposé à cette base peut être compris comme une mélodie en deux couches, toutes deux descendantes diatoniquement ${ }^{21}$, et l'interversion des figures rythmiques entre les deux premières mesures présentées (mordant/blanche, puis blanche/mordant) lui confère un effet polyphonique particulier ${ }^{22}$. Un dernier élément à considérer est le trille suivi d'un mordant sur les degrés 1-7-1 comme conclusion du passage, procédé qui constitue également une allusion au baroque du fait de l'utilisation de son ornementation caractéristique. Ce passage donne naissance à une mélodie sobre, qui entretient de subtils rapports avec le tango, et une écriture qui favorise une atmosphère baroque.

Dans le second exemple, on identifie une écriture contrastante à la contrebasse, avec la proposition d'un marcato en uno tres comprenant des arrastres, et une levée chromatique typique, éléments qui suggèrent un passage renfermant un important swing de tango. La mélodie n'est absolument plus séparable en couches, mais complètement linéaire, avec tous ses déplacements conjoints. On constate également qu'elle est bien plus rythmique, et qu'elle comporte aussi un fort rapport avec le tango :

21 Dans cette lecture, la première couche va du mi au premier temps jusqu'au do à la troisième mesure, tandis que la seconde commence au si au troisième temps et va jusqu'au so/de la troisième mesure.

22 On obtient ainsi une mélodie qui alterne mordant en couche grave/blanche en couche aiguë/blanche en couche grave/mordant en couche aiguë/ blanche en couche grave, en conférant cet effet polyphonique à la mélodie, car les différentes couches et figurations semblent alors se répondre, effet favorisé par le décalage rythmique entre ces réponses. 
un commencement acéphale à la deuxième mesure présentée, une répétition de ce début en décalage qui favorise un rythme asymétrique, et sa continuation à la troisième mesure avec une phrase caractéristique du tango : acéphale, avec un lié vers la deuxième croche, la troisième croche en staccato et la quatrième accentuée, ce qui rend la phrase asymétrique, et prolongée par la suite par des rythmes dactyle et anapeste.

On peut observer la façon dont Piazzolla utilise, dans un même morceau, un même matériau - harmonie et motif mélodique - en cherchant à l'exploiter de deux façons différentes, premièrement en clé de baroque, puis en clé de tango. C'est dans le contraste du traitement musical de ces deux passages que réside cette stratégie visant à articuler ces univers sonores: le métissage avec changement de clé de lecture23. Considérons désormais comment cette articulation proposée par Piazzolla semble également être très délicatement mise en œuvre.

Une articulation au dosage très précis

Revenons-en, pour commencer, à l'exposition de la fugue à la figure 3, dont le sujet présente par ailleurs d'importantes caractéristiques mélodiques du tango (Marsili, 2012, p. 86 ; López, Sonia, 2008, p. 138), en articulant ainsi éléments baroques et de tango. Cette articulation se dévoile également dans la présence d'un effet sonore caractéristique du tango, le tambor24, réalisé au violon, tout au long de cette exposition de la fugue, et dont aussi bien l'aspect timbrique que les rythmes réalisés constituent d'importantes références au tango 25 . Si l'on prend en

\footnotetext{
23 L'on peut être tenté de penser qu'en réalisant une juxtaposition de ces deux univers musicaux, en les exposant l'un après l'autre, le compositeur n'effectue pas une articulation directe entre eux, l'utilisation du même matériau ayant pour fonction tout à la fois de donner une unité à la pièce et de montrer les différences entre les deux clés proposées. Néanmoins, les articulations observées à l'intérieur de chacune de ces sections nous conduisent plutôt à voir une recherche d'une combinaison entre les éléments de tango et ceux baroques tout au long de la composition : ces changements de clé de lecture proposent plutôt un point de vue différent pour apprécier ces articulations qu'une utilisation d'un même matériau dans deux styles distincts.

24 Pour plus de détails, voir Mauriño (2008, p. 22).

25 Les coups sur les deuxième et quatrième temps peuvent être saisis comme une référence au yumbeado; le rythme qui suit, entre les mesures 7 et 12, peut être
} 
considération que la présence d'une percussion est un élément étranger à la sonorité de la fugue (Pelinski, 2008, p. 46), on se rend compte de l'introduction d'un élément du tango dans cette exposition de la fugue. D'un autre côté, cette percussion proposée par Piazzolla semble également répondre au souci de ne pas marquer excessivement cette partie de la fugue : au début de la composition, pendant l'entrée en solo du bandonéon, le violon ne réalise que deux coups par mesure, et assure cette fonction rythmique de façon discrète; à deux voix on observe le violon réaliser quatre coups par mesure, et à trois voix sept, ce qui amène à constater que ce rythme, tout en accompagnant le crescendo des entrées des voix qui s'accumulent, correspond également au souci de ne pas occuper trop de place par rapport à l'exposition de la fugue qui est en train de se dérouler. On perçoit ainsi une volonté de doser de façon précise les éléments employés : on pourrait même imaginer le compositeur se dire «tango oui, mais pas trop : il s'agit aussi d'une fugue baroque ».

Le choix des instruments constitue une autre donnée intéressante. Piazzolla décide de commencer cette exposition avec le bandonéon. Cet instrument est peut-être le plus emblématique du tango, et le rapport avec ce genre est donc mis en évidence par ce choix ; néanmoins, sa référence à la sonorité de l'orgue peut également suggérer un lien avec la musique baroque dans son timbre. Il est également très intéressant de noter que le deuxième instrument à réaliser son entrée est précisément la guitare électrique, dont la sonorité propose un rapport avec le jazz'26 (Brunelli, 2011). La troisième entrée est effectuée par une doublure de la contrebasse avec le piano qui, comme nous l'avons vu, représente aussi une façon caractéristique d'accompagner dans le tango. On constate ainsi que le seul instrument qui ne propose pas le sujet de fugue est le violon, et si l'on considère qu'il s'agit d'un instrument très caractéristique et

compris comme une variation du 3-3-2, en ayant pour rythme (2)-3-3, tandis que le dernier rythme semble justement proposer une variante du 3-3-2.

26 Piazzolla a vécu une partie importante de son enfance et adolescence à New York, et a été un grand amateur de jazz tout au long de sa vie. 
emblématique de la musique baroque, on se demande si ne pas profiter de cette référence pendant l'exposition de la fugue vise un objectif particulier.

Une hypothèse fictive peut nous aider à répondre à cette question. Si le compositeur intervertissait le rôle du violon et celui de la guitare électrique, le premier instrument réalisant l'une des apparitions du sujet, et le second les marquages rythmiques, le résultat timbrique aurait été le rapprochement avec une fugue baroque par la réalisation du sujet au violon, instrument qui est caractéristique de cette période historique. Toutefois ces marquages rythmiques, réalisés à la guitare électrique et sans l'importante référence timbrique de l'effet tambor au violon, perdraient sans doute une grande partie de ce qui les apparente au tango. Comme on le voit, le choix du dosage des divers éléments dans ces articulations paraît assez délicat, et il nous semble observer une recherche d'un juste équilibre entre les éléments qui proposent une sonorité baroque, et ceux qui renvoient au tango. Il est donc possible de penser que dans cette exposition de la fugue suggérant de très importantes références au baroque, le compositeur propose, par la présence de cet accompagnement rythmique au violon aussi bien que par l'absence de son timbre à l'une des entrées du sujet, de renforcer les liens avec le tango dans ce passage. On constate la précision subtile avec laquelle Piazzolla cherche à mélanger ces divers éléments ; il lui faut la juste dose d'éléments et de sonorités faisant référence au baroque, mais il doit également ajouter d'autres éléments dans une très juste mesure afin d'atteindre une sonorité qui, tout en y faisant référence, puisse également s'en démarquer d'une certaine façon, pour créer un style qui lui sera particulier, et ce notamment en conservant la référence du tango.

Dans cette exposition l'utilisation de blue notes, élément caractéristique du jazz, est également susceptible de nous renseigner sur ce dosage précis des courants musicaux articulés. 
Figure 8 - Astor Piazzolla. Muerte del ánge/(s.d.). Bandonéon, m. 27-31, $\pm \cong 140$, vers 0 ' 34 , partition manuscrite par Bragato, mesure à $4 / 4$

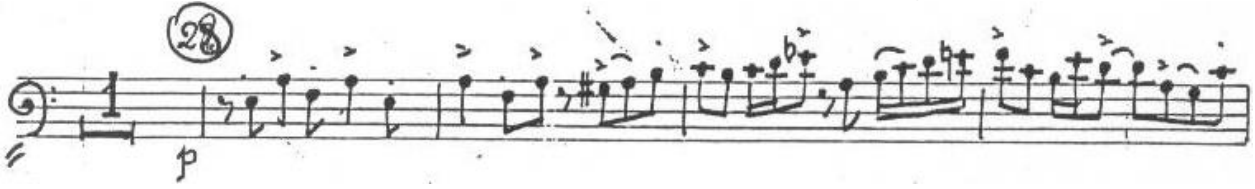

À la quatrième mesure exposée, l'on observe la présence d'un mib, quinte diminuée de la tonalité de la mineur, que nous pouvons considérer comme une blue note (Cugny, 2009, p. 239-240). Ce passage est extrait du thème, lors de sa réalisation en homophonie; il est intéressant de constater que cette même phrase musicale, réalisée lors de l'exposition de la fugue, est proposée sans cette blue note, comme on le constate à la neuvième mesure présentée ci-dessous.

Figure 9 - Astor Piazzolla. Muerte del ánge/ (s. d.). Bandonéon, m. 1-10, $\pm \cong 142$, partition manuscrite par Bragato

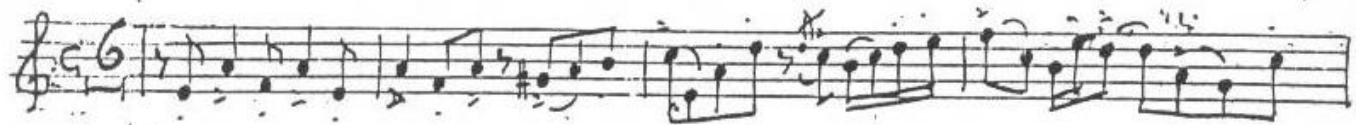

Il est donc possible de supposer, en se basant sur cette différence d'écriture, que Piazzolla considère cette blue note comme une sonorité non adéquate à la fugue, puisqu'il préfère éviter cette note caractéristique dans la construction de son sujet 27 . Il est temps d'observer d'autres passages de cette même pièce.

Figure 10 - Astor Piazzolla. Muerte del ánge/ (s. d.). Bandonéon et guitare électrique, mesures $7-8, \pm \cong 142$, vers 0 '09, retranscription des mesures 13-14 du manuscrit de Bragato, mesure à 4/4

27 Nous considérons cette donnée même si le compositeur remplace ce passage avec la blue note par deux sauts de quarte juste consécutifs, un élément qui se démarque aussi quelque peu, bien que moins ostensiblement, de la sonorité baroque qui semble recherchée à ce moment-là. 


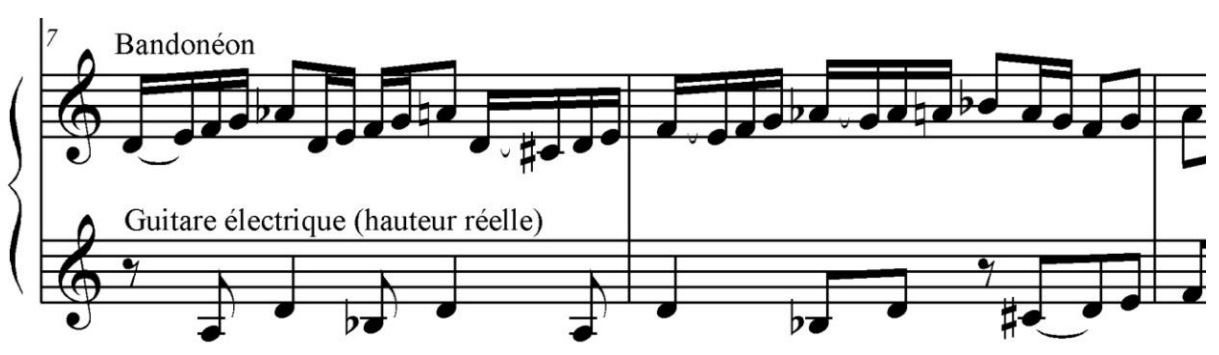

Dans ce fragment de l'exposition de la fugue, temporairement en ré mineur, nous identifions à deux reprises des quintes diminuées 28 dont la résolution va par la suite être réalisée sur la quinte juste29, et que nous pouvons également considérer comme une sonorité de blue note ${ }^{30}$. Etant donné qu'il s'agit d'un contre-sujet, au moment de l'exposition suivante du sujet celles-ci réapparaissent, ces blue notes se faisant entendre sur un autre instrument ${ }^{31}$.

Après avoir observé ces données, une question se pose. Pourquoices notes caractéristiques du jazz sont-elles les bienvenues, dans cette même exposition de la fugue, dans le contre-sujet, alors qu'elles semblent avoir été chassées du sujet? II est peut-être possible de l'expliquer par le fait que la présence d'une blue note dans le thème principal de l'exposition de la fugue, joué en solo, représenterait une référence excessivement explicite au jazz, dans un contexte où ce sont plutôt les éléments baroques et du tango qui semblent être mis en avant. L'introduction de ces blue notes dans le contre-sujet, qui est lui-même toujours joué en même temps que le sujet de la fugue, et le fait que ce dernier ne contienne

28 Les lab au deuxième temps de la septième mesure et au deuxième temps de la huitième mesure au bandonéon.

29 Les la naturels au deuxième temps de la septième mesure et au deuxième temps de la huitième mesure.

30 Le premier cas s'avère un peu plus emblématique, puisque dans le deuxième cette quinte juste (/a naturel) servira de note de passage pour atteindre ensuite le sibémol; de toute façon, le compositeur reviendra dans ce deuxième cas sur le la naturel en tant que note cible au tout début de la mesure suivante, ce qui renforcera le caractère de blue note en y trouvant sa résolution à la quinte juste.

31 Aux mesures 13 et 14 de la figure 3 , on peut voir le contre-sujet réalisé par la guitare électrique dans la tonalité de sol mineur, les blue notes (réb) étant présentes aux deuxièmes temps de ces deux mesures. 
pas de blue note, permettent à ces notes caractéristiques de se glisser dans le contexte général, en y offrant une sonorité distincte, mais sans toutefois y proposer une sonorité qui fasse clairement et franchement référence au jazz, ce qui est une façon d'introduire cet élément tout en le laissant au second plan. II nous semble ainsi possible de penser, à partir de ces analyses, que le compositeur porte un soin assez spécial à l'incorporation d'éléments d'origines diverses, en dosant, dans ce cas précis, la juste mesure de la combinaison des matériaux du jazz, du baroque et du tango. Cette utilisation judicieuse permet de penser que le compositeur dispose les divers éléments en couches, en mettant au premier plan ceux qui lui semblent les plus appropriés, et en laissant dans les couches intermédiaires les éléments dont il souhaite la présence, maisd'une manière plus subtile. Piazzolla semble ainsi adapter son matériau musical, le mouler de façon à le rendre plus confortable dans le contexte d'une exposition de fugue. Et selon ce que l'on peut aussi constater, il ne se limite pas à s'adapter à la fugue ; les divergences avec l'écriture traditionnelle, telles que l'utilisation de l'alternance de rythmes binaires et ternaires ${ }^{32}$ dans le sujet ou par le déroulement d'une exposition de fugue modulante ${ }^{33}$, montrent bien qu'il prétend lui imposer des structures différentes, ou étrangères ${ }^{34}$. II semble donc que l'enjeu consiste à trouver un juste équilibre entre l'utilisation d'un langage et l'introduction dans celui-ci de données nouvelles, originales, propres à la musique de Piazzolla, et comme il est possible de le remarquer grâce à ces analyses, il s'agit là d'un équilibre très sensible et auquel le compositeur semble accorder un soin particulier.

32 Dans son Traité de la fugue, André Gedalge recommande : « [...] l'on évitera avec soin, dans la fugue d'école [...] les sujets renfermant une alternance de rythmes binaires et ternaires » (Gedalge, s.d., p. 9, règle 11).

33 Cette exposition de fugue à la figure 3 est à modulations successives à distance de quarte juste, la première entrée du sujet étant en la mineur, la deuxième en ré mineur (m. 7), la troisième en sol mineur (m. 13).

34 Cette structure modulante doit fort probablement son origine à la musique savante moderne de Béla Bartók, qui est présente dans la partition de Musique pour instruments à cordes, percussion et célesta et sans doute connue de Piazzolla (Saavedra, 1989 ; Sottile, 2007). 


\section{Conclusion}

Nous avons observé comment les éléments baroques sont fortement imprégnés du tango à travers l'utilisation, souvent simultanée, de ses modèles d'accompagnement, de ses phrasés caractéristiques, de ses sonorités typiques, qui engendrent ainsi une unité musicale. Ces éléments exogènes peuvent ainsi se dissoudre dans la production sonore de Piazzolla. En paraphrasant une déclaration de Brunelli concernant à l'origine les aspects caractéristiques du jazz dans la musique de Piazzolla, l'on peut affirmer que ce compositeur incorpore des traits jusqu'alors externes au tango à la trame de son écriture de telle sorte qu'ils s'entrelacent pour pratiquement disparaître absorbés dans le langage tanguistique; et même si les éléments externes au tango ne sont pas toujours aussi complètement absorbés, l'image proposée par ce chercheur est éclairante. C'est comme si le tango de Piazzolla était capable d'assimiler, d'engloutir, d'intégrer et d'incorporer ces éléments, qui s'ajoutent à sa musique sans engendrer pour autant une perte de l'identité du tango, mais en lui conférant ainsi cohérence et unité. De cette manière, c'est la préservation de cette identité qui constitue un important élément d'unification du métissage proposé par Piazzolla, la centralité du tango permettant d'absorber les caractéristiques émanant des autres cultures musicales tout en conservant une cohésion et une cohérence musicales. On peut donc suggérer que le compositeur conçoit son dialogue entre ces courants musicaux comme une expansion de son tango, pour paraphraser Gabriela Mauriño. Le tango de Piazzolla est ainsi capable d'assimiler certains éléments de la musique baroque et de s'en enrichir en les incorporant, en les englobant, et en les interprétant en termes de tango ${ }^{35}$,

35 «Piazzolla s'approprie des musiques trouvées dans ses pérégrinations en les réinterprétant en termes de tango : recours du jazz - walking bass, batterie, improvisation -, du pop - instruments électroniques et percussion -, du rock - sa "sonorité excitante et agressive, nouvelle et romantique" (Piazzolla) -, sans oublier des techniques de la musique savante - passages atonals, ostinato, "basse de malagueña", texture contrapuntique, échelles octaphoniques, etc., se plient à son style. " "Piazzolla se apropia de las músicas encontradas en sus peregrinaciones al reinterpretarlas en términos de tango: recursos del jazz - walking bass, batería, 
la synthèse stylistique de ce musicien étant conçue « structurellement et esthétiquement à partir du territoire du tango »36. Cette recherche d'une identité pour sa musique, que nous avons pu dégager des analyses musicales, s'avère par ailleurs extrêmement fréquente dans ses déclarations, dans un discours où est fortement présente l'intention de produire une musique qui reflète sa ville et son pays (Ferrer, 1980, p. 754 ; Thiers, 1999, p. 403 ; Saavedra, 1989 ; López, Luciano, 1993, p. 114 ; Piazzolla, Astor, 1992 ; Gorin, 1990, p. 79).

Outre la centralité du tango, nous avons également observé une autre stratégie d'articulation, le métissage avec changement de clé de lecture, où l'on identifie une exploitation du degré d'intensité de chaque courant musical articulé. Ainsi, en ce qui concerne la variation de cette intensité dans les diverses parties d'une même composition l'on constate une sorte d'illumination réciproque des courants musicaux employés, le tango proposant une nouvelle lumière au baroque, et le baroque dévoilant une face différente du tango. Il convient d'observer, à chaque changement d'intensité, la mise en valeur proposée de cette articulation elle-même, et l'on peut penser que l'une des fonctions de ces métissages consiste à mettre le tango sous de nouveaux éclairages, à partir de cette interaction avec les autres musiques.

Effectivement, si Piazzolla semble chercher à préserver l'identité du tango, il faut également souligner une volonté substantielle d'y introduire des nouveautés. On perçoit ainsi dans sa musique qui propose cette articulation constante de divers univers, de même que dans ses déclarations (Thiers, 1999, p. 400 ; López, Luciano, 1993, p. 112 ; Saavedra, 1989), une intention d'établir un dialogue entre le tango et ces autres sphères musicales. Ceci nous amène aussi à penser qu'était

improvisación -, del pop - instrumentos electrónicos y percusión -, del rock - su "sonoridad excitante y agresiva, nueva y romántica" (Piazzolla)-, sin olvidar técnicas de la música erudita - pasajes atonales, ostinato, "bajo de malagueña", textura contrapuntística, escalas octofónicas, etc., se pliegan a su estilo. "Pelinski (2008, p. 45-46).

36 “[...] la síntesis estilística que allí logra está diseñada estructural y estéticamente a partir del territorio tanguero." Pelinski (2008, p. 47). 
recherchée une certaine quête de légitimité, résultant du contact du tango avec la musique savante (Lahire, 2015, p. 108-109), ici représentée par la musique baroque, ce qui peut également être perçu dans ses déclarations (Gorin, 1990, p. 79 ; Piazzolla, Diana, 2002, p. 136 ; Cantando, 1957).

En outre, nous avons également observé que cette articulation est souvent réalisée par le biais d'une écriture élaborée et complexe. En effet, il a été possible de percevoir la manière dont les traits de ces univers culturels sont dosés avec une précision très grande dans sa musique, ce qui dévoile une proposition esthétique bien définie dans laquelle ces éléments de diverses cultures sont soigneusement mis en relation, ainsi combinés d'une manière particulièrement rigoureuse.

\section{Références}

BRUNELLI, Omar García. La obra de Astor Piazzolla y su relación con el tango como especie popular urbana. Revista del Instituto de Investigación Musicológica “Carlos Vega”, Buenos Aires, № 12, p. 155-221, 1992.

BRUNELLI, Omar García. La incorporación del jazz a la trama del tango en la música de Astor Piazzolla [audio]. Colloque International Tango: Créations, Identification, Circulations. Ecole des Hautes Etudes en Sciences Sociales \& Maison des cultures du monde, Paris, 2011. $<$ http://globalmus.net/?Communications-audio-mp3\&lang=fr $>$ $<07 / 03 / 13>$.

Cantando La revista de la música popular. Année 1, № 31 . Buenos Aires: Editorial Emilio Roca, 5/11/1957.

CLEMENTE, Peter Arthur. The Structural and Cyclical Organization of Astor Piazzolla's Las Cuatro Estaciones Porteñas. Thèse (Doctor of musical arts) - University of Hartford, 2012.

CUGNY, Laurent. Analyser le jazz. Paris: Outre mesure, 2009.

FERRER, Horacio. El libro del tango - Tomo / Crónica del tango. Buenos Aires: Antonio Tersol, 1980.

FISCHERMAN, Diego et GILBERT, Albert. Piazzolla el mal entendido. Buenos Aires: Edhasa, 2009. 
GEDALGE, André. Traité de la Fugue. 1ère partie, De la Fugue d'Ecole. Paris : Enoch et Cie éditeurs, s.d.

GOLDENBERG, Gabriela. Aproximación al procedimiento fugado en Astor Piazzolla [texte envoyé personnellement par la chercheuse]. Primeras Jornadas de Investigación Astor Piazzolla. CC. San Martín, 2003.

GORIN, Natalio. Astor Piazzolla / A Manera de Memorias. Buenos Aires: Editorial Atlantida, 1990.

KUTNOWSKI, Martín. Rubato instrumental y estructura de la frase en la música de Astor Piazzolla. In: BRUNELLI, Omar García (org.). Estudios sobre la obra de Astor Piazzolla. Buenos Aires: Gourmet Musical Ediciones, 2008, p. 99-108.

LAHIRE, Bernard. Ceci n'est pas qu'un tableau. Essai sur l'art, la domination, la magie et le sacré. Paris: Éditions La Découverte, 2015.

LÓPEZ, Luciano Londoño. Astor Piazzolla, la leyenda [Entrevista com Astor Piazzolla]. Revista universidad de Antioquia, Medellín, volume LXII, numéro 232, p. 111-116, 1993.

LÓPEZ, Sonia Alejandra. Fuga y misterio de Astor Piazzolla. Acercamiento a una "fuga tanguificada". In: BRUNELLI, Omar García (org.). Estudios sobre la obra de Astor Piazzol/a. Buenos Aires: Gourmet Musical Ediciones, 2008, p. 133-143.

MARSILI, Andrea. Potentialité de mutation du tango rioplatense. Bornes du tango porteño et réseaux d'interconnexion avec le tango nomade. Thèse (doctorat en musique) - Université Paris-Sorbonne, 2012.

MAURIÑO, Gabriela. Raíces tangueras de la obra de Astor Piazzolla. In: BRUNELLI, Omar García (org.). Estudios sobre la obra de Astor Piazzolla. Buenos Aires: Gourmet Musical Ediciones, 2008, p. 19-33.

PELINSKI, Ramón. Astor Piazzolla: entre tango y fuga, en busca de una identidad estilística. In: BRUNELLI, Omar García (org.). Estudios sobre la obra de Astor Piazzolla. Buenos Aires: Gourmet Musical Ediciones, 2008, p. 35-56.

PERALTA, Julián. La orquesta típica - Mecánica y aplicación de los fundamentos técnicos del tango. $2^{\text {a }}$ ed. Buenos Aires: Editorial de Puerto, 2012. 
PIAZZOLLA, Astor. "Nuestro tiempo » [Texte de la pochette de l'album]. Piazzolla, Astor et Quintette «Nuevo Tango». Nuestro tiempo [CD]. Argentine : Sony BMG/Columbia, 2005 [LP sorti à l'origine en 1962].

PIAZZOLLA, Astor. Entrevista [réalisée par sa fillle Diana Piazzolla]. La maga, Buenos Aires, año 1, № 26, 08/07/1992.

PIAZZOLLA, Diana. Astor. Anglet: Atlántica, 2002.

SAAVEDRA, Gonzalo. Entrevista con Astor Piazzolla. Barcelona, 1989. $<$ http://www.piazzolla.org/interv/ $><16 / 07 / 2013>$.

SALGÁN, Horacio. Curso de tango. $3^{\text {a }}$ ed. Buenos Aires: A Fuego Lento, 2012.

SOTTILE, Antonieta. «Entretien avec Gerardo Gandini : "Dans l'Argentine d'aujourd'hui, la composition est devenue une chose solitaire" ». Circuit : musiques contemporaines, vol. 17, $\mathrm{n}^{\circ} 2,2007$, p. 73-81. $<$ http://www.erudit.org/revue/circuit/2007/v17/n2/016841 ar.pdf $><19$ /02/2016>.

SPERATTI, Alberto. Con Piazzolla. Buenos Aires: Editorial Galerna, 1969.

THIERS, Walter. El jazz criollo y otras yerbas. Buenos Aires: Corregidor, 1999.

Partitions

BACH, J.-S. Toccata et fugue [BWV 565]. s.d. $<$ http://www.lespartitions.info/gratuites/partitions/pdf/bach-toccataet-fugue-bwv565.pdf $><14 / 01 / 2014>$.

PIAZZOLLA, Astor. La mufa. Buenos Aires: Editorial Lagos, 1974.

PIAZZOLLA, Astor. Mar del Plata 70. Buenos Aires: Editorial Lagos, 1974.

PIAZZOLLA, Astor. Muerte del ángel [partition manuscrite par José Bragato]. s. d. <http://leroncosad.blogspot.fr/2011/10/astor-piazzollala-muerte-del-angel.html $><4 / 11 / 2013>$.

PIAZZOLLA, Astor. Resurrección del ángel. Buenos Aires: Melos, 2002.

Phonographie

CORONATO, Alberto. "Sin retorno ». Astor Piazzolla et son Quintette « Nuevo Tango ». Nuestro tiempo [CD]. Argentine : BMG Ariola Argentina S.A., 2005 [réédition de LP Sony Music Entertainment CBS 8351 de 1962). 
DELFINO, Enrique P. et ROMERO, Manuel ; DELFINO, Enrique P. et LINNING, Samuel. «Medley : Recuerdo de bohemia / Milonguita ». Astor Piazzolla et son «Nouvel octette ». Tango contemporáneo [CD]. Argentine: Sony BMG/Ariola, 2005 [réédition de LP Sony Music Entertainment CBS 9039 de 1963).

MANZI, Homero et PIANA, Sebastián. « Milonga triste ». Astor Piazzolla et son Quintette «Nuevo Tango ». Nuestro tiempo [CD]. Argentine: Sony BMG Ariola, 2005 [réédition de LP CBS 8351 de 1962].

PIAZZOLLA, Astor. "La mufa ». Astor Piazzolla et son Quintette « Nuevo Tango ». Concierto de tango en el Philharmonic Hall de New York [MP3]. Universal, 2005 (réédition de LP POLYDOR 27136, Argentine de 1965). 